\title{
Facet joint changes after application of lumbar nonfusion dynamic stabilization
}

\author{
Soo Eon Lee, MD, ${ }^{1}$ Tae-Ahn Jahng, MD, PhD,2,3 and Hyun Jib Kim, MD, PhD² \\ ${ }^{1}$ Department of Neurosurgery, Seoul National University Hospital; ${ }^{2}$ Seoul National University and Bundang Hospital; and ${ }^{3}$ Seoul \\ National University College of Medicine, Seoul, Korea
}

\begin{abstract}
OBJECTIVE The long-term effects on adjacent-segment pathology after nonfusion dynamic stabilization is unclear, and, in particular, changes at the adjacent facet joints have not been reported in a clinical study. This study aims to compare changes in the adjacent facet joints after lumbar spinal surgery.

METHODS Patients who underwent monosegmental surgery at $L 4-5$ with nonfusion dynamic stabilization using the Dynesys system (Dynesys group) or transforaminal lumbar interbody fusion with pedicle screw fixation (fusion group) were retrospectively compared. Facet joint degeneration was evaluated at each segment using the CT grading system.

RESULTS The Dynesys group included 15 patients, while the fusion group included 22 patients. The preoperative facet joint degeneration CT grades were not different between the 2 groups. Compared with the preoperative CT grades, 1 side of the facet joints at L3-4 and L4-5 had significantly more degeneration in the Dynesys group. In the fusion group, significant facet joint degeneration developed on both sides at L2-3, L3-4, and L5-S1. The subjective back and leg pain scores were not different between the 2 groups during follow-up, but functional outcome based on the Oswestry Disability Index improved less in the fusion group than in the Dynesys group.

CONCLUSIONS Nonfusion dynamic stabilization using the Dynesys system had a greater preventative effect on facet joint degeneration in comparison with that obtained using fusion surgery. The Dynesys system, however, resulted in facet joint degeneration at the instrumented segments and above. An improved physiological nonfusion dynamic stabilization system for lumbar spinal surgery should be developed.

http://thejns.org/doi/abs/10.3171/2015.10.FOCUS15456
\end{abstract}

KEY WORDS facet joint degeneration; nonfusion; dynamic stabilization; adjacent-segment pathology

$\mathrm{T}$ HE facet joints of the lumbar spine are recognized as major stabilizing structures in the aging spine and a source of low-back pain. . $, 5,9,22$ As the intervertebral discs degenerate with age, stress on the facet joints increases and this leads to facet joint changes such as hypertrophy, arthritis, and bony spur formation. In rigid fixation of the lumbar spine using pedicle screws, the fused segment increases the stress on the adjacent segments by preventing motion in the fused segment. This affects the intervertebral discs and facet joints of the adjacent segments. After rigid fixation, such stress accelerates degenerative changes and contributes to the development of adjacent-segment pathology (ASP). Furthermore, facet joints, especially the upper facet joint, are affected by pedicle screw insertion, which has been regarded as a risk factor for ASP in lumbar instrumented fusion. . $3,13,17,21^{2}$
Recently, nonfusion dynamic stabilization systems have been applied to the management of degenerative lumbar spinal disease, and several studies have shown favorable clinical and radiological outcomes. ${ }^{6,10,18,20}$ Despite the theoretical advantages over rigid fixation, the long-term effect of nonfusion dynamic stabilization on ASP has been disputed.7 Although some authors have documented ASP development after nonfusion stabilization, there are no clinical reports on facet joint changes at the stabilized and adjacent segments after nonfusion stabilization.

Therefore, a comparison of facet joint degeneration after nonfusion dynamic stabilization and rigid fixation is needed to determine the effectiveness of nonfusion stabilization. Herein, we report a clinical and radiological comparative study on facet joint degeneration following fusion and nonfusion surgeries.

ABBREVIATIONS ASP = adjacent-segment pathology; COR = center of rotation; FEM = finite elements modeling; ODI = Oswestry Disability Index; PSF = pedicle screw fixation; ROM = range of motion; TLIF = transforaminal lumbar interbody fusion; VAS = visual analog scale.

SUBMITTED September 1, 2015. ACCEPTED October 21, 2015.

INCLUDE WHEN CITING DOI: 10.3171/2015.10.FOCUS15456. 


\section{Methods \\ Patient Population}

Both transforaminal lumbar interbody fusion (TLIF) with pedicle screw fixation (PSF) and nonfusion dynamic stabilization using the Dynesys system (Zimmer Spine) were performed between 2003 and 2010 at our institution. The operative indications were degenerative disc disease with radiculopathy, central or foraminal stenosis, degenerative spondylolisthesis, and degenerative lumbar scoliosis. A retrospective study was planned to compare changes in the facet joints after lumbar spinal surgery. Patients who underwent surgery for degenerative lumbar spinal disease at L4-5 were divided into 2 groups: TLIF with PSF (fusion group), and nonfusion dynamic stabilization using the Dynesys system (Dynesys group). In both groups, the patients who underwent CT of the lumbar spine at a minimum of 12 months after the operation were included. Patient exclusion criteria were spinal instability from lumbar spinal trauma, previous lumbar fusion surgery, and severe osteoporosis under medical treatment.

\section{Surgical Procedure}

All operations were performed with the patient in a neutral prone position by 1 senior surgeon. In the fusion group, a conventional or minimally invasive TLIF procedure was used. For minimally invasive TLIF, the surgeon used bilateral $2.5-\mathrm{cm}$ skin incisions and a self-designed tubular retractor system. Laminectomy, facetectomy, discectomy, and interbody fusion were performed through an 18- to $20-\mathrm{mm}$ diameter tubular retractor. Pedicle screw insertion and rod fixation were performed through a tubular retractor with fluoroscopic guidance. In the Dynesys group, conventional posterior or bilateral mini-open Wiltse approaches were used. In some patients, a $2.5-\mathrm{cm}$ midline skin incision was made to allow decompression. Screw insertions were performed using the bilateral miniopen technique. To preserve facet joint integrity, care was taken to place the Dynesys screw lateral to the facet joints.

\section{Radiological Evaluation}

Facet joint degeneration was evaluated bilaterally at each segment (L1-2, L2-3, L3-4, L4-5, and L5-S1), except L4-5 was evaluated postoperatively in the fusion group due to the performance of the uni- or bilateral facetectomies. Degeneration was classified as Grades 0 to 3 based on previously reported CT criteria: Grade 0 , normal facet joint space (2-4 mm); Grade 1, narrowing of the joint space $(<2 \mathrm{~mm})$, small osteophytes, and/or mild articular process hypertrophy; Grade 2, narrowing of the joint space $(<2 \mathrm{~mm})$, moderate osteophytes, articular process hypertrophy, and/or mild subarticular bone erosions; and Grade 3, narrowing of the joint space $(<2 \mathrm{~mm})$, severe osteophytes, articular process hypertrophy, severe subarticular bone erosions, and/or subchondral cysts. ${ }^{23}$ Determination of the changes in the facet joints was based on differences between the preoperational and final postoperative CT scan. On the final evaluated CT scan, facet joint violation was based on the screw or the connecting rod being in line with, as well as abutting on or into, the L3-4 and L4-5 facet joint. ${ }^{21}$ The radiological data were investigated using a picture archiving and communication system (PACS; M-view, version 5.483; Infinitt Healthcare).

\section{Clinical Evaluation}

Subjective back and leg pain levels were assessed using the visual analog scale (VAS). Functional outcome was assessed using the Oswestry Disability Index (ODI). Clinical parameters were measured preoperatively, postoperatively at 12, 24, and 36 months, and at the final follow-up. In addition, neural intervention and medication via pain analgesics were evaluated at the final follow-up.

\section{Statistical Analysis}

Independent t-tests or Wilcoxon signed-rank tests were used to evaluate continuous variables, and the chi-square test or Fisher exact test was used to evaluate categorical variables. A linear mixed model was used to test differences in the clinical parameters. Statistical significance was defined as a $\mathrm{p}$ value $<0.05$. Analyses were performed using SPSS (version 19.0; IBM).

\section{Results}

Between 2003 and 2010, 29 patients underwent TLIF with PSF at L4-5 and 23 patients underwent Dynesys at L4-5. Among these patients, 22 and 15 patients who underwent CT scanning 12 months after surgery were included in the fusion and Dynesys groups, respectively (Table 1). The fusion group had a mean age of $60.86 \pm$ 8.34 years and included 11 male and 11 female patients. The Dynesys group had a mean age of $58.33 \pm 7.03$ years and included 6 male and 9 female patients. Preoperatively, all patients had lumbar degenerative disease and exhibited lumbar spinal stenosis associated with spondylolisthesis and herniated intervertebral disc. The final CT evaluation was conducted at $30.68 \pm 21.34$ months in the fusion group and at $24.93 \pm 15.34$ months in the Dynesys group $(\mathrm{p}=$ 0.57). The mean total clinical follow-up period was 61.56 \pm 23.56 months in the fusion group and $51.15 \pm 25.56$ months in the Dynesys group ( $\mathrm{p}=0.78)$.

\section{Changes in the Facet Joints on CT}

The preoperative and final follow-up CT-based grades of the facet joint degeneration on both the right and left sides are presented in Fig. 1. Preoperative facet joint degeneration between the 2 groups did not differ at all levels $(p>0.05)$. Preoperative facet joint degeneration was greatest at L4-5 in both groups. At the final CT evaluation, facet joint degeneration had significantly progressed at all segments except L1-2 in the fusion group (Fig. 1 left). In the Dynesys group, increased degeneration was detected at the instrumented segment facet joint (L4-5) and the above facet joint (L3-4), but the difference was significant only on 1 side of each segment (Fig. 1 right).

Facet joint violation was detected in 4 screws in 2 patients (13.6\%) in the Dynesys group: 2 screws (1 on each side) at L3-4 and 2 left-side screws at L4-5. However, in the fusion group, 36 screws violated the facet joints in 20 patients (90.9\%): 10 right-side screws and 15 left-side screws at L3-4, 4 right-side screws and 7 left-side screws 
TABLE 1. Patient demographics*

\begin{tabular}{lccc}
\hline \multicolumn{1}{c}{ Variable } & Fusion Group $(\mathrm{n}=22)$ & Dynesys Group $(\mathrm{n}=15)$ & $\mathrm{p}$ Value \\
\hline Sex (male/female) & $11: 11$ & $6: 9$ & 0.74 \\
\hline Mean age (yrs) & $60.86 \pm 8.34$ & $58.33 \pm 7.03$ & 0.47 \\
\hline Preoperative diagnosis & & & 0.94 \\
\hline Lumbar stenosis & 7 & 6 & \\
\hline Lumbar stenosis w/ spondylolisthesis & 14 & 2 & 0.57 \\
\hline Lumbar stenosis w/ HIVD & 1 & $24.93 \pm 15.34$ & 0.78 \\
\hline Mean time to last CT evaluation (mos) & $30.68 \pm 21.34$ & $51.15 \pm 25.56$ & \\
\hline Mean clinical follow-up period (mos) & $61.56 \pm 23.56$ & & \\
\hline HIVD $=$ herniated intervertebral disc. \\
$*$ Mean values are presented as the mean \pm SD.
\end{tabular}

at L4-5. The changes in facet joint degeneration in both groups are illustrated in Figs. 2 and 3.

\section{Clinical Outcomes}

The clinical parameters in terms of VAS and ODI were not preoperatively different between the 2 groups (all $\mathrm{p}>$ 0.05 ). The VAS values for back and leg pain significantly decreased during the follow-up period $(\mathrm{p}<0.05)$, and there was no difference in the back and leg pain scores between the 2 groups $(p=0.620$ and $p=0.190$, respectively; Fig. $4 \mathrm{~A}$ and $\mathrm{B})$. The mean ODI score significantly decreased during the follow-up period in the Dynesys group. In the fusion group, the mean ODI score decreased after TLIF, but gradually increased after the the 24-month follow-up period (Fig. 4C). A statistically significant difference in the ODI score was observed between the 2 groups during the follow-up period $(\mathrm{p}=0.006)$. At the final follow-up, 12 patients in the fusion group had taken nonopioid and/ or opioid medications, and 4 of those patients additionally underwent neural intervention, including nerve root block and medical facet block. At the final follow-up, 7 patients in the Dynesys group had taken pain analgesics, mainly nonopioids.

Six years after TLIF surgery, 1 patient underwent vertebroplasty at L-2 and L-3. Additional surgery for ASP at L3-4 or L5-S1 was recommended to 4 patients in the fusion group and 1 patient in the Dynesys group; however, these patients did not undergo the additional surgery, and their ASP was controlled through conservative management.

\section{Discussion}

The objective of nonfusion dynamic stabilization is to maintain the normal physiological range of motion (ROM) and reduce disc and facet joint stress. Biomechanically, the Dynesys system preserves ROM and the center of rotation (COR) closer to that of the intact spine than obtained using rigid fixation..$^{4,15,19,24}$ The theoretically advantageous biomechanical properties of the nonfusion approach suggest that it should produce better clinical outcomes, especially regarding ASP prevention. Successful clinical outcomes in patients with nonfusion dynamic stabilization have been reported, but definitive clinical or radiological benefits compared with conventional instrumented fusion have not been clearly demonstrated. Nonfusion dynamic stabilization using the Dynesys system is reported to have a positive effect on the prevention of ASP development, but other authors have not reported such advantages over fusion surgery. Thus, the long-term effect on ASP after Dynesys implantation is not clear, and its effect on adjacent facet degeneration has been reported only in biomechanical studies..$^{10,12,14,16,18,20}$

In this study, we conducted the first clinical investigation into facet joint changes after the application of a nonfusion dynamic stabilization system with a clinical follow-up period of over 4 years and a radiological followup period of over 2 years. The results of our study show that, compared with TLIF surgery, Dynesys application can significantly reduce the incidence of lumbar spine facet joint degeneration and provide functional improvement. Facet joint degeneration was observed at 2 levels above, 1 level above, and 1 level below the adjacent segments in the fusion group, but in the Dynesys group facet joint degeneration was confined to the stabilized segment and the adjacent above segment. In addition, functional outcomes during follow-up improved less in the fusion group than in the Dynesys group. The Dynesys group showed less frequent facet joint degeneration at the adjacent segments, but significant facet joint degeneration was detected at the instrumented segment. Interestingly, although facet joint degeneration at the instrumented segment was accelerated in the Dynesys group, facet joint violation rarely developed intraoperatively. This implies that Dynesys can have an undesirable effect on the instrumented segment facet joint. This supports the results of a finite elements modeling (FEM) study that revealed increased facet contact force after Dynesys implantation. ${ }^{7}$

Several researchers have investigated facet joint contact force in nonfusion dynamic stabilization, and the Dynesys system is reported to produce a greater facet contact force than that in the normal spine under all loading conditions. ${ }^{11,14,16}$ A biomechanical FEM-based study of the Dynesys system at L4-5 showed that the facet contact force during extension increased with an increase in moment. ${ }^{7}$ This undesirable effect on facet contact force would contribute to facet joint degeneration at the instrumented segment. In addition, it might contribute to the development of unusual postoperative back pain; that is, pain that differs from the usual postoperative back pain due to op- 

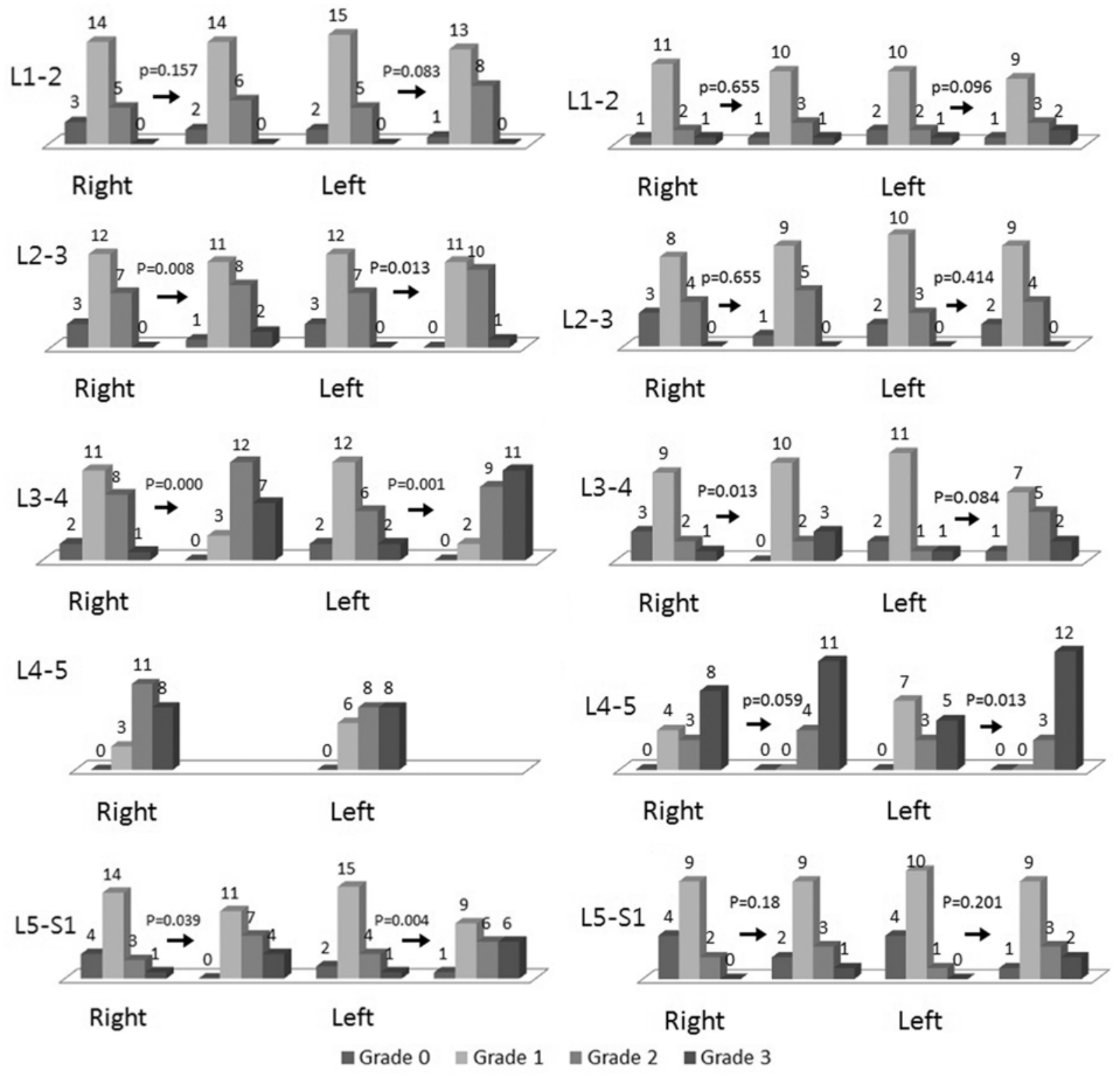

FIG. 1. Facet joint changes on CT. Left: In the fusion group, facet joint degeneration at the final CT evaluation was present in the 2 levels above (L2-3), 1 level above (L3-4), and 1 level below (L5-S1). There were statistically significant increases in degeneration on both the right and left sides. Right: In the Dynesys group, facet joint changes on CT were not observed in the second and third levels above (L1-2), 2 levels above (L2-3), and 1 level below (L5-S1). However, facet joint degeneration developed at the level above instrumentation (L3-4) as well as the instrumented level itself (L4-5). There were statistically significant changes only in the right L3-4 and left L4-5 segments. Grades refer to the CT grades of facet joint degeneration on the right and left sides.

erative trauma or soft-tissue injury. This is similar to postoperative back pain after lumbar artificial disc replacement, which increases facet joint stress. In addition to its effects on facet contact force, several biomechanical studies on the Dynesys system have revealed nonphysiological biomechanical effects, such as insufficient ROM and abnormal instantaneous COR in comparison with the intact spine. ${ }^{15,19,24}$ Other authors have reported that the stiffness that results from Dynesys stabilization is the same as that from rigid fixation. ${ }^{7,8}$ All of these studies suggest that the Dynesys system is limited when attempting to simulate the normal spinal motion of an intact spine. In our study, Dynesys application led to facet joint degeneration at the adjacent segment, but the degree of degeneration and its distribution among the other adjacent segments were not as great as that in the fusion group. Thus, our results suggest that nonfusion stabilization using the Dynesys system can reduce or prevent ASP to some extent. The biomechanical discrepancies and design characteristics of the Dynesys system may impact ASP development, but it is not as great as that from TLIF.

In addition, we found a higher incidence of facet joint degeneration and more unsatisfactory clinical outcomes in the fusion group than those in the Dynesys group. Facet joint violation developed in the fusion group and facet joint degeneration was demonstrated, not only in the violated 

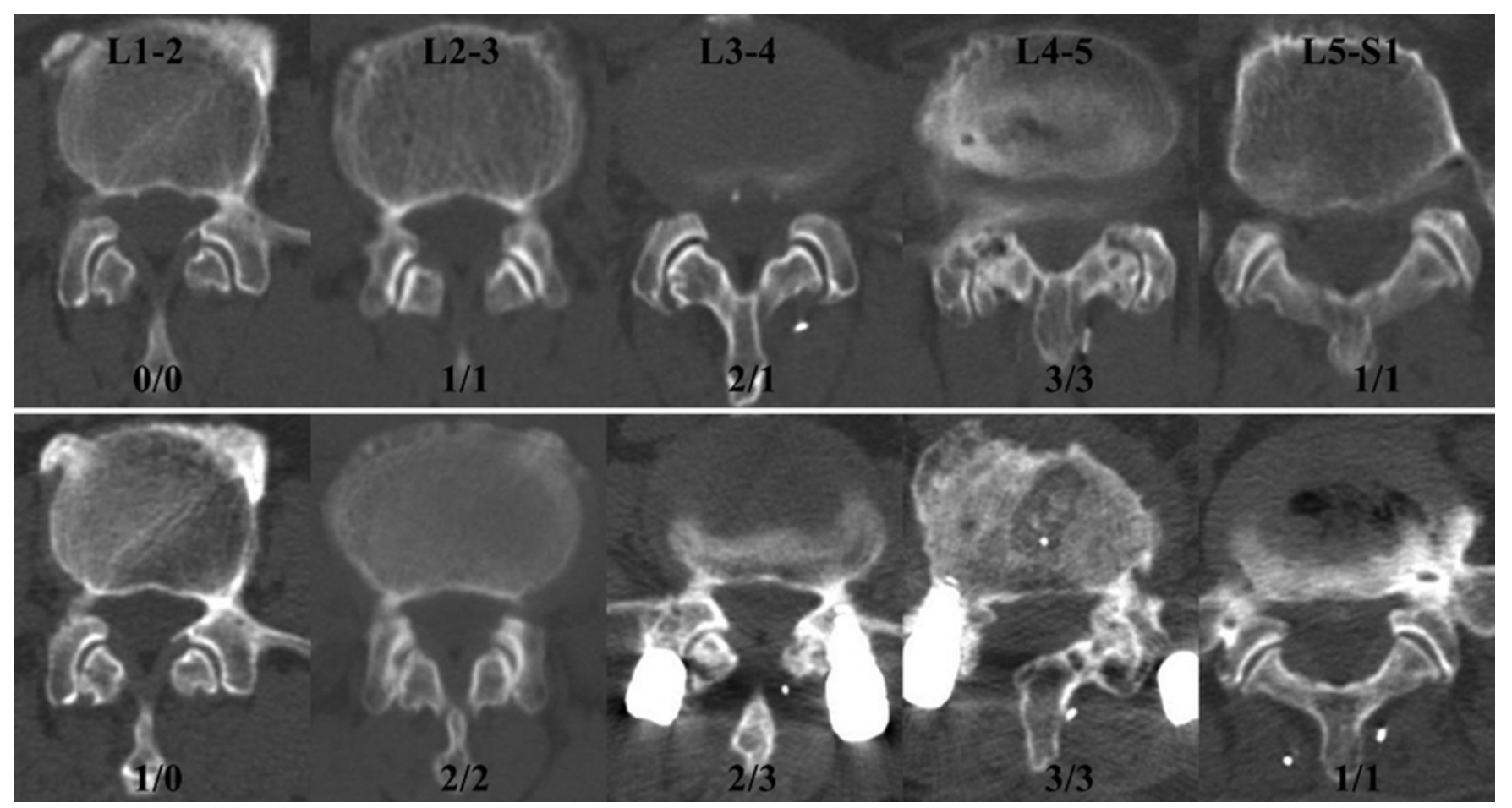

FIG. 2. Example of facet joint degeneration in the fusion group. A 65-year-old woman underwent TLIF with PSF surgery at L4-5 due to spinal stenosis. Upper: Preoperative CT grades for facet joint degeneration. Lower: CT grades at 24 months postoperatively. Preoperatively, the facet joint at L4-5 was severely degenerated, but other segments showed mild to moderate grade changes. At the final CT, facet joint violation was observed at both L3-4 and left L4-5. Right L4-5 facetectomy was identified, and facet degeneration had progressed from the preoperative level, including 1 level above the segment (L2-3).

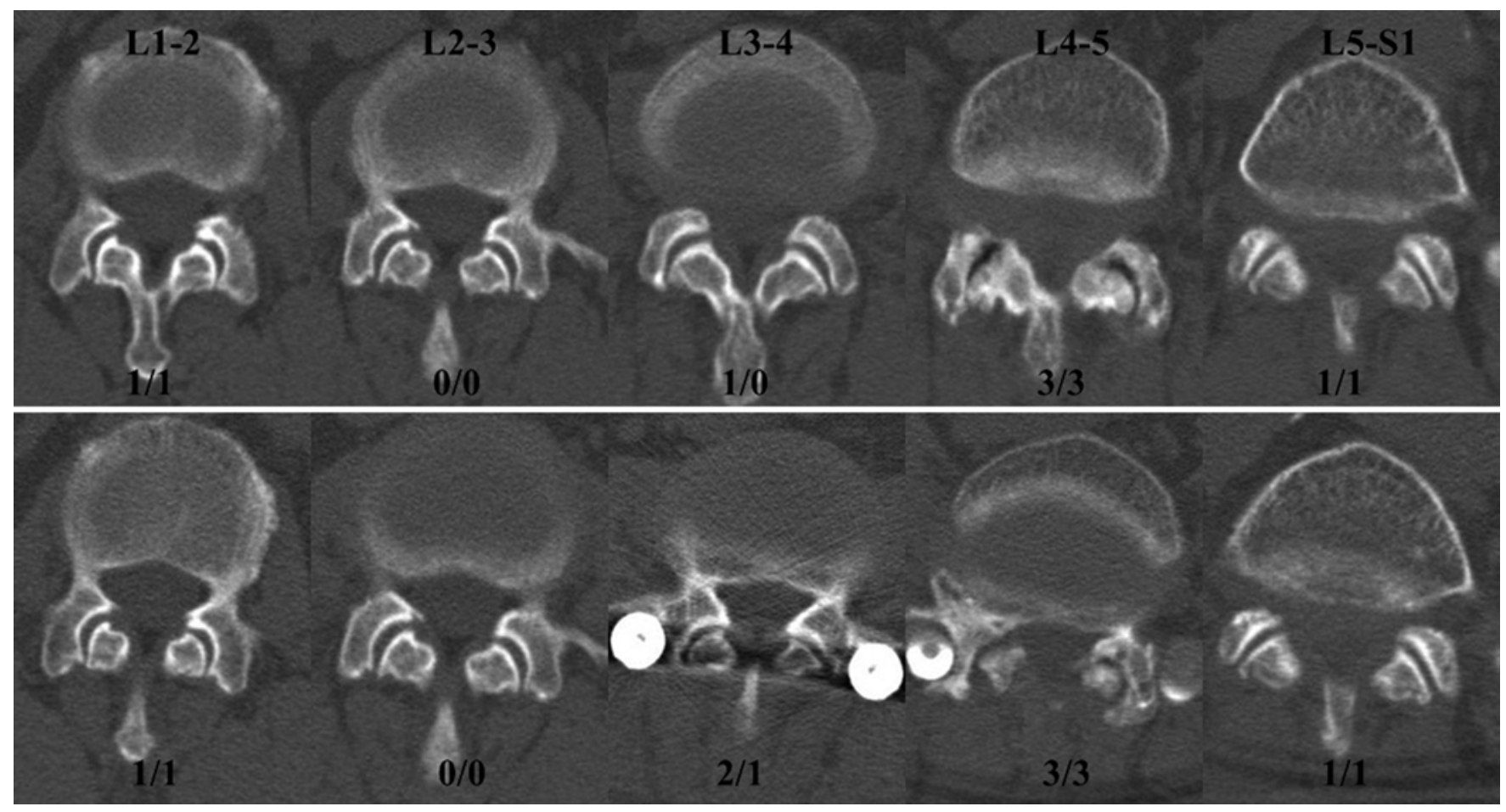

FIG. 3. Example of facet joint changes in the Dynesys group. A 69-year-old woman underwent nonfusion dynamic stabilization surgery using the Dynesys system. Upper: Preoperative CT grades for facet degeneration. Lower: CT grades at 36 months postoperatively. Preoperatively, the facet joint at L4-5 was severely degenerated, but the other segments had normal to mild CT grades. Postoperatively, facet joint violation at $L 3-4$ and $L 4-5$ was not demonstrated, but adjacent facet joint degeneration at L3-4 progressed. Postoperative and preoperative CT grades were the same at the other segments (L1-2, L2-3, and L5-S1). 

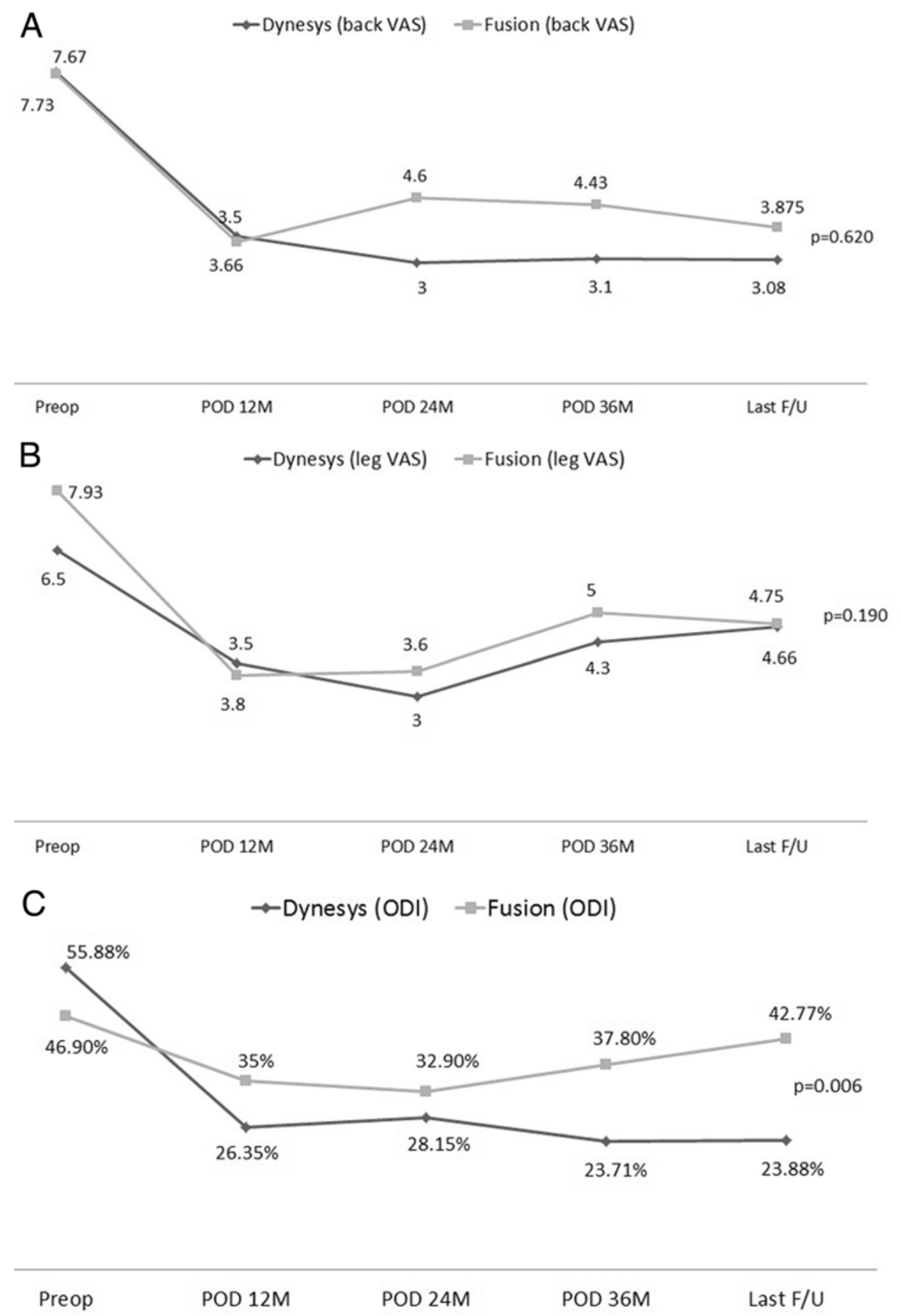

FIG. 4. Clinical outcomes. A: VAS for back pain significantly decreased between preoperation and the final follow-up. There was no statistically significant difference in the back pain VAS levels between the fusion and Dynesys groups during the postoperative course $(p=0.620)$. B: VAS for leg pain also decreased postoperatively. There was no significant difference in leg pain VAS levels between the 2 groups during the postoperative course $(p=0.190)$. C: Functional outcome, as represented by ODI. The linear mixed model showed a statistically significant difference between groups. In the fusion group, ODI improved less than in the Dynesys group $(p=0.006)$. F/U = follow-up; POD = postoperative duration.

segment (L3-4) but also in the 2 segments above (L2-3) and 1 segment below (L5-S1). In addition, while VAS for back and leg pain decreased postoperatively, functional outcomes gradually deteriorated and half of the TLIF pa- tients needed analgesics or neural intervention at the final evaluation. Therefore, we suggest that fusion surgery has long-term clinical and radiological drawbacks related to accelerated facet joint degeneration. 
There are several limitations in the present study. This study represents a single surgeon's experience at 1 institution; hence, it involved a small number of patients-15 Dynesys and 22 fusion patients-resulting in low statistical power. Lumbar stenosis with spondylolisthesis comprised a large portion of the preoperative diagnoses, but the small number of patients did not allow a subgroup analysis. The mean follow-up time of 24 to 30 months might be insufficient to identify radiological ASP. Although the levels of facet joint degeneration in our 2 groups were not preoperatively different, facet joint degeneration at the segments above and below the instrumented segment were greater in the fusion group than in the Dynesys group; however, the difference was without statistical significance. The mean final CT evaluation period was longer by 6 months in the fusion group, and this might have provided more time for the development of ASP. Facet joint violation was also more common in the fusion group and a contributing factor for ASP. If ASP is the result of a natural aging process or the result of fusion surgery, the type of underlying degeneration might lead to more rapid degeneration. Therefore, our results may not be solely due to the type of surgery. Patients were advised to visit the outpatient clinic at regular postoperative periods $(1,3,6$, and 12 months and every subsequent year). Regardless, CT evaluation was not prospectively planned at those regular follow-up periods; therefore, our comparative study could not include regular CT images. As a similar limitation of retrospective studies, preoperative MRI of the lumbar spine was evaluated in all patients but postoperative MRI was not regularly planned. If facet joint degeneration was evaluated on both CT and MRI, a more exact evaluation could be conducted. Although theoretical background studies on nonfusion dynamic stabilization have indicated its utility in maintaining normal physiological ROM and COR and reducing disc and facet joint stress, the Dynesys system did not provide comprehensive advantages over fusion stabilization. Improvements in nonfusion dynamic stabilization systems may result in better clinical and radiological outcomes for the treatment of degenerative lumbar spinal disease.

\section{Conclusions}

Compared with fusion surgery, nonfusion dynamic stabilization was advantageous as it reduced or prevented facet joint degeneration and improved clinical outcomes. The Dynesys system, however, did not prevent facet joint degeneration at the instrumented and adjacent segments. Improvements in nonfusion dynamic stabilization systems are needed.

\section{References}

1. Boswell MV, Colson JD, Sehgal N, Dunbar EE, Epter R: A systematic review of therapeutic facet joint interventions in chronic spinal pain. Pain Physician 10:229-253, 2007

2. Cardoso MJ, Dmitriev AE, Helgeson M, Lehman RA, Kuklo TR, Rosner MK: Does superior-segment facet violation or laminectomy destabilize the adjacent level in lumbar transpedicular fixation? An in vitro human cadaveric assessment. Spine (Phila Pa 1976) 33:2868-2873, 2008

3. Chen Z, Zhao J, Xu H, Liu A, Yuan J, Wang C: Technical factors related to the incidence of adjacent superior segment facet joint violation after transpedicular instrumentation in the lumbar spine. Eur Spine J 17:1476-1480, 2008

4. Cunningham BW, Gordon JD, Dmitriev AE, Hu N, McAfee PC: Biomechanical evaluation of total disc replacement arthroplasty: an in vitro human cadaveric model. Spine (Phila Pa 1976) 28:S110-S117, 2003

5. Datta S, Lee M, Falco FJ, Bryce DA, Hayek SM: Systematic assessment of diagnostic accuracy and therapeutic utility of lumbar facet joint interventions. Pain Physician 12:437-460, 2009

6. Di Silvestre M, Lolli F, Bakaloudis G, Parisini P: Dynamic stabilization for degenerative lumbar scoliosis in elderly patients. Spine (Phila Pa 1976) 35:227-234, 2010

7. Jahng TA, Kim YE, Moon KY: Comparison of the biomechanical effect of pedicle-based dynamic stabilization: a study using finite element analysis. Spine J 13:85-94, 2013

8. Kim CH, Chung CK, Jahng TA: Comparisons of outcomes after single or multilevel dynamic stabilization: effects on adjacent segment. J Spinal Disord Tech 24:60-67, 2011

9. Le Huec JC, Basso Y, Aunoble S, Friesem T, Bruno MB: Influence of facet and posterior muscle degeneration on clinical results of lumbar total disc replacement: two-year follow-up. J Spinal Disord Tech 18:219-223, 2005

10. Lee SE, Park SB, Jahng TA, Chung CK, Kim HJ: Clinical experience of the dynamic stabilization system for the degenerative spine disease. J Korean Neurosurg Soc 43:221-226, 2008

11. Liu CL, Zhong ZC, Shih SL, Hung C, Lee YE, Chen CS: Influence of Dynesys system screw profile on adjacent segment and screw. J Spinal Disord Tech 23:410-417, 2010

12. Morishita Y, Ohta H, Naito M, Matsumoto Y, Huang G, Tatsumi M, et al: Kinematic evaluation of the adjacent segments after lumbar instrumented surgery: a comparison between rigid fusion and dynamic non-fusion stabilization. Eur Spine J 20:1480-1485, 2011

13. Moshirfar A, Jenis LG, Spector LR, Burke PJ, Losina E, Katz JN, et al: Computed tomography evaluation of superior-segment facet-joint violation after pedicle instrumentation of the lumbar spine with a midline surgical approach. Spine (Phila Pa 1976) 31:2624-2629, 2006

14. Niosi CA, Wilson DC, Zhu Q, Keynan O, Wilson DR, Oxland TR: The effect of dynamic posterior stabilization on facet joint contact forces: an in vitro investigation. Spine (Phila Pa 1976) 33:19-26, 2008

15. Niosi CA, Zhu QA, Wilson DC, Keynan O, Wilson DR, Oxland TR: Biomechanical characterization of the three-dimensional kinematic behaviour of the Dynesys dynamic stabilization system: an in vitro study. Eur Spine J 15:913-922, 2006

16. Rohlmann A, Burra NK, Zander T, Bergmann G: Comparison of the effects of bilateral posterior dynamic and rigid fixation devices on the loads in the lumbar spine: a finite element analysis. Eur Spine J 16:1223-1231, 2007

17. Roy-Camille R, Roy-Camille M, Demeulenaere C: [Osteosynthesis of dorsal, lumbar, and lumbosacral spine with metallic plates screwed into vertebral pedicles and articular apophyses.] Presse Med 78:1447-1448, 1970 (Fr)

18. Schaeren S, Broger I, Jeanneret B: Minimum four-year follow-up of spinal stenosis with degenerative spondylolisthesis treated with decompression and dynamic stabilization. Spine (Phila Pa 1976) 33:E636-E642, 2008

19. Schmoelz W, Huber JF, Nydegger T, Dipl-Ing, Claes L, Wilke HJ: Dynamic stabilization of the lumbar spine and its effects on adjacent segments: an in vitro experiment. J Spinal Disord Tech 16:418-423, 2003

20. Schnake KJ, Schaeren S, Jeanneret B: Dynamic stabilization in addition to decompression for lumbar spinal stenosis with degenerative spondylolisthesis. Spine (Phila Pa 1976) 31:442-449, 2006 
21. Shah RR, Mohammed S, Saifuddin A, Taylor BA: Radiologic evaluation of adjacent superior segment facet joint violation following transpedicular instrumentation of the lumbar spine. Spine (Phila Pa 1976) 28:272-275, 2003

22. Trouillier H, Kern P, Refior HJ, Müller-Gerbl M: A prospective morphological study of facet joint integrity following intervertebral disc replacement with the CHARITÉ Artificial Disc. Eur Spine J 15:174-182, 2006

23. Weishaupt D, Zanetti M, Boos N, Hodler J: MR imaging and CT in osteoarthritis of the lumbar facet joints. Skeletal Radiol 28:215-219, 1999

24. Zander T, Rohlmann A, Burra NK, Bergmann G: Effect of a posterior dynamic implant adjacent to a rigid spinal fixator. Clin Biomech (Bristol, Avon) 21:767-774, 2006

\section{Disclosures}

The authors report no conflict of interest concerning the materi- als or methods used in this study or the findings described in this paper. No benefits in any form have been or will be received from any commercial party related directly or indirectly to the subject of this manuscript.

\section{Author Contributions}

Conception and design: Jahng. Acquisition of data: Lee. Analysis and interpretation of data: Lee. Drafting the article: Lee. Critically revising the article: Jahng, Kim. Reviewed submitted version of manuscript: Jahng. Approved the final version of the manuscript on behalf of all authors: Jahng. Statistical analysis: Lee. Study supervision: Jahng, Kim.

\section{Correspondence}

Tae-Ahn Jahng, Department of Neurosurgery, Seoul National University Bundang Hospital, Seoul National University College of Medicine, 173-82 Gumi-Ro, Bundang-Gu, Seongnam, Gyeonggi-Do 463-707, Republic of Korea. email: taj@snu.ac.kr. 